\title{
Analysis of Gene Expression in a Gut Epithelial Cell Line Treated with re:iimmune and Lipopolysaccharide
}

Keywords: Dietary supplement; Inflammation; Gene expression; Nutrigenetics; Lipopolysaccharide; Ginger extract; Zinc oxide

\begin{abstract}
Background: Re:iimmune is a novel oral hydration and probiotic supplement designed to provide clinical strength hydration and intestinal immune support following illness and hospitalization. The goal of this study was to evaluate any anti-inflammatory properties of the supplement due to the inclusion of ginger extract and zinc oxide in the product formulation.
\end{abstract}

Methods: A gut epithelial cell line was stimulated using lipopolysaccharide (LPS) before the addition of the re:iimmune product to the culture medium. Following incubation, gene expression arrays were used to assay 92 different markers involved in a wide-variety of inflammatory and anti-inflammatory processes. Additionally, ten hallmark pro-inflammatory proteins were assayed using immunofluorescent imaging.

Results: Findings from qPCR assays indicate that five inflammatory markers were significantly altered as a result of incubation with the re:iimmune product, including the two genes involved in the synthesis and binding of prostaglandins. Immunofluorescent techniques revealed that the re:iimmune product was capable of attenuating LPS-induced inflammation in hallmark pro-inflammatory proteins, including TNF-a and NF-KB.

Conclusions: While the mechanism remains unclear, the results from this study indicate that the inclusion of zinc and ginger extract in the formulation of the re:iimmune product may serve to reduce cellular inflammation through the attenuation of pro-inflammatory cytokines.

\section{Abbreviations}

RI: re:iimmune; LPS: Lipopolysaccharide; RT-qPCR: Quantitative reverse transcription polymerase chain reaction

\section{Introduction}

Inflammation is an essential biological response that functions to both protect and repair the body in response to physical, biological, chemical, and psychological stimuli. However, excess and chronic inflammation can be very damaging to an organism. In addition to unambiguous inflammatory disorders such as asthma and lupus, inflammation has also been linked to a multitude of complex disorders including cancer, cardiovascular disorders, and Alzheimer's disease $[1,2]$.

Given the negative outcomes associated with excess and chronic inflammation, more attention is now being devoted to development and use of anti-inflammatory agents. The best known class of antiinflammatory agents is the non-steroidal anti-inflammatory drugs (NSAID) pain relievers. However, prolonged use of these medications

\section{Journal of}

Nutrition and Health

\section{Rhy Norton*, Austin O'Reilly and David Miley}

Dynamic DNA Laboratories, 2144 E Republic Rd, Ste. b204, Springfield, MO 65804, USA

\section{*Address for Correspondence}

Rhy Norton, Dynamic DNA Laboratories, 2144 E Republic Rd, Ste. b204, Springfield, MO 65804, USA, Tel: (417) 319-1047; E-mail: rhy@dynamicdnalaboratories.com

Submission: 26 July, 2016

Accepted: 19 August, 2016

Published: 24 August, 2016

Copyright: (c) 2016 Norton R et al. This is an open access article distributed under the Creative Commons Attribution License, which permits unrestricted use, distribution, and reproduction in any medium, provided the original work is properly cited.

has been implicated with a multitude of side-effects, including gastric erosions and kidney damage [3]. Due to these dangers, NSAIDs are not suitable for routine ingestion and other, more natural options should be explored for repetitious anti-inflammatory therapies.

Aside from pharmaceutical drugs, a wide variety of foods, herbs and minerals have been shown to have anti-inflammatory qualities. Of these, ginger (Zingiber offinale Roscoe) is one of the best-studied natural compounds and is widely consumed throughout the world. The multitude of health benefits attributed to ginger is thought to be a result of several volatile oils produced by the plant. The most prevalent and well-studied of these compounds is [6]-gingerol (1-[4'-hydroxy3'-methoxyphenyl]-5-hydroxy-3-decanone), but several other volatile organic compounds are also produced by the plant, such as zingerone and shogaols [4]. Given the complex chemical composition of ginger, it is difficult and costly to assay each volatile compound individually. For this reason, no effort was made in this study to differentiate the bioactive properties of the individual components of ginger. Rather, the complex composition of ginger is experimentally treated as a single compound.

The reported anti-inflammatory effects of ginger are numerous and several mechanisms of action have been proposed. One of the most prevalent explanations for the anti-inflammatory abilities of ginger is related to the ability of the compound to inhibit prostaglandin biosynthesis [5]. In addition, ginger has proven to inhibit the release of nitric oxide and the production of pro-inflammatory cytokines following exposure to interferon- $\gamma$ and lipopolysaccharide (LPS) [6].

Zinc supplementation has also been shown to act as an antioxidant and anti-inflammatory treatment in healthy human subjects. The inclusion of zinc in the diet is of great concern as the World Health Organization (WHO) have estimated that up to 2 billion individuals worldwide may be deficient in zinc. For this reason, many processed foods have started adding zinc oxide during manufacturing as a source of the element. Zinc is thought to function as an antiinflammatory through the regulation of reactive oxygen species (ROS), subsequently inhibiting NF- $\mathrm{KB}$ activation and associated targets such as TNF- $\alpha$ and IL- $1 \beta$ [7]. 
Citation: Norton R, O’Reilly A, Miley D. Analysis of Gene Expression in a Gut Epithelial Cell Line Treated with re:iimmune and Lipopolysaccharide. J Nutri Health. 2016;2(2): 6.

In light of this evidence, a dietary supplement has been developed to naturally reduce inflammation through the inclusion of ginger extract and zinc oxide in the product formulation. Re:iimmune (RI) is a combination dietary supplement that is designed to decrease inflammation through the prevention of dehydration and introduction of natural compounds with beneficial nutraceutical properties, largely modulated through the addition of ginger extract and zinc oxide. One serving of RI consists of the following: zinc oxide (10 mg), chloride (1,710 mg), sodium (890 mg), potassium (380 mg), L-glutamine $(6,000 \mathrm{mg})$, ginger extract $(275 \mathrm{mg})$, inulin (1,000 mg), and a blend of probiotic microorganisms totaling $3.4 \times 10^{13} \mathrm{CFU}$ at the time of manufacturing. This mixture supplies the potential for antiinflammatory benefits to the user via three different mechanisms: 1) Supplementation with ginger extract and zinc oxide, 2) Prevention of dehydration, and 3) Stabilization of the gut microbiota through the use of prebiotic fibers and probiotic microorganisms.

In this study, only the cellular anti-inflammatory properties of RI were tested using an LPS-induced inflammation model in a well-established gut epithelial cell line. This model is well suited to assay anti-inflammatory agents at the cellular level and is an ideal method to investigate the therapeutic effects of the zinc and ginger extract contained in the RI product. LPS was used to bind the CD14/ TLR4/MD2 receptor complex and promote the release of proinflammatory cytokines. Following the application of re:iimmune, potential reduction in the cytokines was then assayed using $\mathrm{qPCR}$ and immunocytochemistry.

\section{Materials and Methods}

\section{Cell culture}

HT-29 (ATCC, Manassas, VA) gut epithelial cells were grown to confluency in McCoy's 5A medium (Sigma, St. Louis, MO) with $10 \%$ fetal bovine serum (FBS) (MidSci, Valley Park, MO), $25 \mathrm{mM}$ $\mathrm{NaHCO}_{3}$ (Fisher, Walthman, MA), $100 \mathrm{U} / \mathrm{mL}$ penicillin (Fisher, Walthman, MA), and $100 \mu \mathrm{g} / \mathrm{mL}$ Streptomycin (Fisher, Walthman, MA). Cells were then split and transferred to 6-well culture plates and grown overnight. Test wells were then treated with $3.0 \mu \mathrm{L}$ of a $1 \mathrm{mg} / \mathrm{mL}$ LPS stock 0111: B4 (Sigma, St. Louis, MO) and incubated for 30 minutes. Following LPS treatment, $30 \mu \mathrm{L}$ of a $10 \mathrm{X}$ suspension of RI was added to each well, for a final concentration of $1 / 10$ the recommended product preparation. In certain trials, probiotic microorganisms were removed from the $10 \mathrm{X}$ suspension of RI by passing the suspension through a $0.45 \mu \mathrm{m}$ syringe filter. Wells treated with RI were incubated for 1 hour. A total of four conditions were tested per trial: Control (no treatment), LPS, RI, and LPS+RI.

\section{RNA extraction and cDNA synthesis}

Total RNA was extracted from each group using and Purelink ${ }^{\text {tw }}$ RNA MiniKit (Ambion, Foster City, CA) using Trizol' reagents for lysis and RNA stabilization. RNA was extracted according to manufacturer's protocol, using $3 \times 30 \mu \mathrm{L}$ elutions. RNA was quantified using a NanoDrop ${ }^{\text {Tx }}$ 2000C nanospectrophotometer with typical yields of $\sim 22 \mu \mathrm{g}$ of RNA. Total RNA was converted to cDNA using a High Capacity cDNA Reverse Transcription Kit (Applied Biosystem, Foster City, CA). A total of $1.8 \mu \mathrm{g}$ RNA was added per $20 \mu \mathrm{L}$ reaction. Reactions were setup according to manufacturer's recommendations and incubated in a Veriti ${ }^{\mathrm{ma}}$ thermal cycler.

\section{RT-qPCR and aata analysis}

To perform the RT-qPCR, a total of 25 ng of cDNA was added per $10 \mu \mathrm{L}$ reaction, along with $5 \mu \mathrm{L}$ of a $2 \mathrm{X}$ TaqMan Gene Expression Maser Mix. cDNA was not directly quantified, but assumed using input RNA levels. Thermal cycling was performed using a StepOnePlus ${ }^{\mathrm{Tn}}$ instrument as directed by the manufacturer. mRNA expression was determined using a pre-developed TaqMan Array (Applied Biosystems, Foster City, CA - Cat. 4414205). Using this format, 92 inflammatory markers are simultaneously interogated in a 96-well format. Normalization was achieved through the use of a passive reference dye (ROX) and standardization against four endogenous genes, 18S, GAPDH, GUSB, and HRPT1. Relative quantification was determined using $\triangle \Delta \mathrm{Ct}$ methodology. Thresholds and baselines were set automatically according to software default settings.

\section{Cell culture for immunocytochemistry}

HT-29 gut epithelial cells were grown to confluency in McCoy's 5A medium with $10 \%$ fetal bovine serum (FBS), $25 \mathrm{mM} \mathrm{NaHCO}_{3}, 100 \mathrm{U} /$ $\mathrm{mL}$ penicillin, and $100 \mu \mathrm{g} / \mathrm{mL}$ Streptomycin. Confluent $25 \mathrm{~cm}^{2}$ flasks were split and transferred to four 24-well culture plates containing $12 \mathrm{~mm}$ glass coverslips and left to grow overnight in cell media with $500 \mu \mathrm{L} /$ well. Test wells were then treated with $5.0 \mu \mathrm{L}$ of a $100 \mu \mathrm{g} / \mathrm{mL}$ LPS stock and incubated for 30 minutes. Following LPS treatment, $5 \mu \mathrm{L}$ of a 10X suspension of RI was added to each well, for a final concentration of $1 / 10$ the recommended product preparation. Wells treated with RI were incubated for 1 hour. A total of four conditions were tested per trial: Control (no treatment), LPS, RI, LPS+RI. After completion of the experiment, cells were fixed onto the glass coverslips via 15 minute, $4 \%$ paraformaldehyde (Thermo Scientific, Walthman, MA) incubation followed by $3,1 \mathrm{X}$ PBS washes. The final wash was left on the cells and plates were wraped in parafilm and stored at 4 ${ }^{\circ} \mathrm{C}$ for fluorescent imaging. Both unfiltered and filtered experiments were carried out in two independent trials with immunofluorescent staining conducted in duplicate for each experiment. Three images were acquired for each immunofluorescently labeled coverslip.

\section{Immunocytochemistry and data analysis}

Coverslips containing cells were blocked and permeablized with Donkey Serum (Fisher, Walthman, MA) containing $0.01 \%$ Triton (Fisher, Walthman, MA). All primary antibodies (Abcam, Cambridge, MA) were diluted 1:500 in Donkey Serum and incubated

Table 1: Summary of protein levels following the addition of RI.

\begin{tabular}{|l|l|l|l|}
\hline Gene Symbol & Expression & Characteristics & Category \\
\hline$p-E R K 1 / 2$ & Resolved & Inflammatory & Protein Kinase \\
\hline LL-1B & Resolved & Inflammatory & Cytokine \\
\hline L-10 & No change & Anti-inflammatory & Cytokine \\
\hline IL-15 & Resolved & Viral inflammation & Cytokine \\
\hline$M K P 3$ & No change & Anti-inflammatory & Phosphatase \\
\hline NALP3 & Resolved & Inflammatory & Inflammasome Receptor \\
\hline$N F k B$ & Resolved & Inflammatory & Transcription Factor \\
\hline$P-p 38$ & Resolved & Inflammatory & Protein Kinase \\
\hline$P K A$ & Resolved & Inflammatory & Protein Kinase \\
\hline$T N F a$ & Resolved & Inflammatory & Cytokine \\
\hline
\end{tabular}


Citation: Norton R, O’Reilly A, Miley D. Analysis of Gene Expression in a Gut Epithelial Cell Line Treated with re:iimmune and Lipopolysaccharide. J Nutri Health. 2016;2(2): 6.

ISSN: 2469-4185

Table 2: Gene expression assays contained in the human inflammation array used in this study. Genes in bold represent housekeeping genes used in the assay. Genes that were significantly altered as a result of incubation with RI are underlined.

\begin{tabular}{|c|c|c|c|}
\hline $18 S$ & HPRT1 & KLKB1 & PLA2G7 \\
\hline A2M & $\mathrm{HRH} 1$ & KNG1 & PLCB2 \\
\hline ADRB1 & $\mathrm{HRH} 2$ & LTA4H & PLCB3 \\
\hline ADRB2 & $\mathrm{HRH} 3$ & LTB4R & PLCB4 \\
\hline ALOX12 & HTR3A & LTB4R2 & PLCD1 \\
\hline ALOX5 & HTR3B & LTC4S & PLCE1 \\
\hline ANXA1 & ICAM1 & MAPK1 & PLCG1 \\
\hline ANXA3 & IL13 & MAPK14 & PLCG2 \\
\hline ANXA5 & IL1R1 & MAPK3 & PTAFR \\
\hline BDKRB1 & IL1R2 & MAPK8 & PTGDR \\
\hline BDKRB2 & IL1RAPL2 & MC2R & PTGER2 \\
\hline CACNA1C & IL1RL1 & NFKB1 & PTGER3 \\
\hline CACNA1D & IL2RA & NOS2 & PTGFR \\
\hline CACNA2D1 & IL2RB & NR3C1 & PTGIR \\
\hline CACNB2 & IL2RG & PDE4A & PTGIS \\
\hline CACNB4 & ITGAL & PDE4B & PTGS1 \\
\hline CASP1 & ITGAM & PDE4C & PTGS2 \\
\hline CD40 & ITGB1 & PDE4D & TBXA2R \\
\hline CD40LG & ITGB2 & PLA2G10 & TBXAS1 \\
\hline CES1 & KLK1 & PLA2G1B & TNF \\
\hline CYSLTR1 & KLK14 & PLA2G2A & TNFRSF1A \\
\hline GAPDH & KLK15 & PLA2G2D & TNFRSF1B \\
\hline GUSB & KLK2 & PLA2G4C & TNFSF13B \\
\hline HPGD & KLK3 & PLA2G5 & VCAM1 \\
\hline
\end{tabular}

on the coverslips for 3 hours. Coverslips were washed 3 times with 0.1\% Tween (Fisher, Walthman, MA) followed by a PBS wash Secondary antibodies (Abcam, Cambridge, MA) were diluted 1:200 in PBS and incubated on the coverslips for 1 hour. Coverslips were washed 3 times with $0.1 \%$ Tween followed by a PBS wash. After completion of immunocytochemistry staining, coverslips were mounted onto glass slides and 4',6-diamidino-2-phenylindole (DAPI) + Vectashield (Vector Laboratories, Burlingame, CA) was added for nuclear staining and to prevent photo-bleaching. Images were acquired using a Zeiss M2 fluorescent microscope. Images were converted to grayscale and the non-stimulated staining areas of cell patches were used to balance each image. Integrated densities were acquired in three independent images by measuring pixel densities in 5 non-overlapping, circular regions of interest (ROI). Background intensity values were obtained from five non-overlapping regions in areas containing no cells, as determined by DAPI, and average values subtracted from ROI. Relative average means were determined for each condition and data reported as the average fold change \pm SEM relative to the average mean level for control conditions that were set equal to one.

\section{Results}

Protein expression assays
Eleven peptides were evaluated via immunofluorescence in the experimental setup. Ten out of the eleven inflammatory markers tested, produced usable data across all experimental and control groups, with 8 proteins responding to LPS and RI, both filtered and unfiltered. An increase in the pro-inflammatory peptides was observed with cells that were stimulated with LPS (Figure 1). Upon treatment of LPS stimulated cells with RI, pro-inflammatory peptide levels resolved to that of control levels (Figure 2). Additionally, RI was added to set of control wells to evaluate the potential inflammatory effects with no observable differences from basal levels. Of the proteins targeted in the proposal, AHR was dropped in the HT-29 cell line due to complete absence of expression (Table 1).

\section{Gene expression assays}

Out of the 92 inflammatory markers tested, a total of 64 were expressed by the HT-29 cell line and produced usable data across all experimental and control groups (Table 2). Of the endogenous controls, $18 \mathrm{~S}$ was dropped from the analysis due to varying levels among treatments. The remaining markers amplified well with $\Delta \mathrm{Ct}$ values ranging from -1.36 to 13.84 . From the 64 usable array markers, five produced a statistically significant change as a result of incubation with the RI product (Table 3). Specifically, mRNA levels of ADRB2, MAPK3, NF- $\kappa B$ and PTGS2 were significantly increased while PTGER2 was significantly decreased as a result (Figure 3). To provide concise results, only markers altered as a result of RI or a combination of RI and LPS were included in these results. Other markers, such as TNF- $\alpha$, were significantly upregulated as a result of exposure to LPS, but were not included if the addition of RI had no effect on the marker (data not shown).

\section{Discussion}

\section{Overview}

In this study, we explored the relationship between cellular inflammation and the RI product. LPS was selected to induce a pro-inflammatory response in a HT-29 gut epithelial cell line as the product is intended to stabilize the gut through supplementation with ginger and zinc. Individually, both ginger and zinc have been shown throughout multiple studies to mediate levels of pro-inflammatory cytokines. However, studies combining these two agents are lacking and the existence of possible synergistic effects is currently unknown. Results from this study indicate that the RI product is capable of altering several inflammatory processes within the HT-29 cell line, and possibly attenuating some pro-inflammatory responses.

\section{Performance of the LPS-Induced HT-29 cell line}

As expected, the addition of LPS produced significant changes in both mRNA and protein levels in the HT-29 cell line. LPS was shown

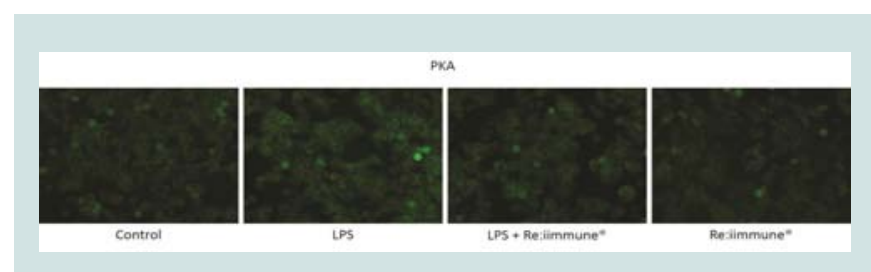

Figure 1: Representative images of immunofluorescent labeled PKA in HT-19 cell culture stimulated with LPS and treated with unfiltered re:iimmune (RI). 
Citation: Norton R, O’Reilly A, Miley D. Analysis of Gene Expression in a Gut Epithelial Cell Line Treated with re:iimmune and Lipopolysaccharide. J Nutri Health. 2016;2(2): 6.

ISSN: 2469-4185

Table 3: Summary of inflammatory genes found to be significantly altered as a result of incubation with re:iimmune $(\mathrm{RI})$.

\begin{tabular}{|l|l|l|l|}
\hline Gene & Expression & Gene Name & Category \\
\hline ADRB2 & Increased & $\begin{array}{l}\text { Adrenergic, beta-2-, receptor, } \\
\text { surface }\end{array}$ & $\begin{array}{l}\text { G-protein coupled } \\
\text { receptor }\end{array}$ \\
\hline MAPK3 & Increased & $\begin{array}{l}\text { Mitogen-activated protein } \\
\text { kinase 3 }\end{array}$ & Protein kinase \\
\hline NFKB & $\begin{array}{l}\text { Increased } \\
\text { with LPS }\end{array}$ & $\begin{array}{l}\text { Nuclear factor of kappa light } \\
\text { polypeptide gene enhancer in } \\
\text { B-cells 1 }\end{array}$ & Transcription factor \\
\hline PTGER2 & Decreased & $\begin{array}{l}\text { Prostaglandin E receptor 2 } \\
\text { (subtype EP2), 53kDa }\end{array}$ & $\begin{array}{l}\text { G-protein coupled } \\
\text { receptor }\end{array}$ \\
\hline PTGS2 & Increased & $\begin{array}{l}\text { Prostaglandin-endoperoxide } \\
\text { synthase 2 (prostaglandin G/H } \\
\text { synthase and cyclooxygenase) }\end{array}$ & Synthase \\
\hline
\end{tabular}

to elevate the pro-inflammatory peptides targeted in this study, while the anti-inflammatory IL-10 and MKP3 remained comparable to controls when stimulated with LPS. These results indicate that LPS acted as successful and selective inflammatory stimuli in a gut epithelial cell line.

\section{Results from immunofluorescent protein expression}

Of the ten proteins evaluated in the immunofluorescence experiments, all 8 of the pro-inflammatory markers displayed elevated levels in the human colon cell line when stimulated with LPS. More importantly, pro-inflammatory marker levels elevated by LPS were attenuated to levels near that of control conditions when treated with RI. While RI restored the pro-inflammatory markers to near control levels, no increase in the two anti-inflammatory peptides was observed. It was also noted that no difference was observed when RI was filtered prior to application, thus eliminating the direct effect of the bacteria in the product. Therefore, we hypothesize that the actionable results observed in our experiment are more likely linked to the effects of ginger extract and zinc oxide contained in the RI. Previous studies have suggested that ginger extract may act against inflammation [8], and more specifically ginger has been shown to suppress TNF- $\alpha$ in human synoviocytes [9]. In addition, [6]-gingerol, a bioactive molecule contained in ginger, was reported to inhibit the production of pro-inflammatory cytokines from LPS-stimulated peritoneal macrophages [10] and suppress NF- $\kappa B$ nuclear activation [11].

\section{Significant changes in gene expression}

This study revealed five genes involved in the inflammatory process to be significantly altered as a result of the addition of RI. Overall, these genes represent a wide variety of different mechanisms including cell signaling, metabolism, and inflammatory responses. The remainder of this discussion will investigate each gene and hypothesize as to any possible link between the therapeutic effects of $\mathrm{RI}$ and known mechanisms of each inflammation-associated marker Table 2 above provides additional information as the inflammationassociated genes identified in this study.

\section{Possible effects on prostaglandin E2 synthesis}

Out of the five genes that were significantly altered as a result of the addition of RI, two were involved in the production or reception of prostaglandin E2 (PGE2). The addition of RI to the medium resulted in the increased expression of PTGS2 (prostaglandin-endoperoxide synthase 2). Often referred to as $C O X-2$, this cyclooxygenases play a key role in the conversion of arachidonic acid into prostaglandin [12]. A significant down regulation of PTGER2 (prostaglandin $\mathrm{E}_{2}$ receptor 2) was observed in the groups receiving only RI. PTGER2 encodes a g-protein coupled receptor, PTGER2 that is responsible for binding PGE2 [13].

Taken together, the altered expression of these two genes linked to prostaglandin synthesis suggests that supplementation with RI is sufficient to alter prostaglandin biosynthesis and/or distribution throughout the body. Indeed, some of the anti-inflammatory effects of ginger have been contributed to its ability to inhibit prostaglandin synthesis [5]. Other studies have supported these findings by demonstrating that [8]-gingerol, one of the many bioactive components of ginger, was capable of inhibiting COX-2 expression [14]. Conversely, findings from this study suggest that the addition of RI results in an increased levels of PGE2 synthesis via COX-2, although the down regulation of PTGER2 receptor suggest that RI may selectively reduce prostaglandin levels in the gastrointestinal tract or in other areas of the body.

When considering the effects on prostaglandin biosynthesis, the inclusion of zinc supplementation also needs to be taken into account with the RI product. Increased levels of zinc have been demonstrated to alter the tissue expression of various prostaglandins within the rat. One study found that zinc supplementation in the rat results in increased PGE2 levels in the plasma and decreased levels in the intestinal tissues [15]. The results from this study are consistent with these findings, suggesting that zinc supplementation is capable of reducing prostaglandin levels in the gut, possibly through the altered expression of PTGER2.

\section{Implications of altered prostaglandin biosynthesis}

The role of prostaglandins in the regulation of inflammatory responses is a highly complex dynamic. Overall, it is generally agreed that prostaglandins (particularly PGE2) are capable of exerting a wide variety of pro-inflammatory and anti-inflammatory responses [16]. These contrasting modes of operation are also known to be mediated by the expression of the receptor gene throughout the tissues of the body. Some of the most notable anti-inflammatory effects of prostaglandins involve PGE2, which has been shown to demonstrate

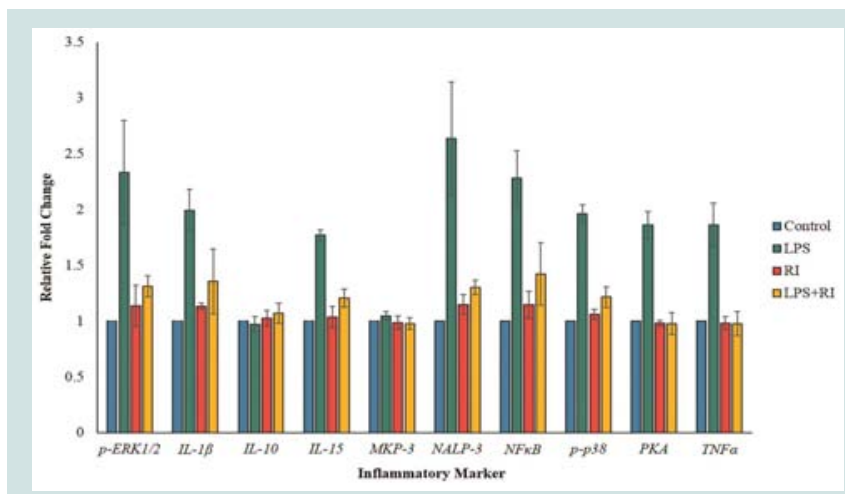

Figure 2: Pro-inflammatory and anti-inflammatory proteins significantly altered by the addition of RI as determined by immunofluorescent microscopy. Error bars represent standard error of the mean. 


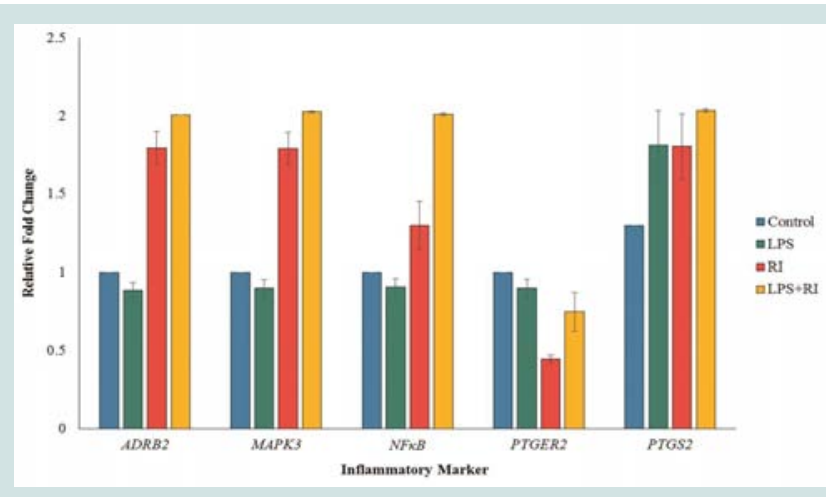

Figure 3: Inflammatory genes significantly altered as a result of the addition of RI as determined by qPCR. Error bars represent standard error of the mean.

a wide variety of effects on both the adaptive and innate immune system. In conjunction with the reduction of pro-inflammatory cytokines observed in the immunofluorescent series of experiments, PGE2 has been shown to exert anti-inflammatory actions on a wide variety of immune cells (i.e. neutrophils, monocytes, natural killer cells), as well as the modulation of Th1 cell differentiation and some B cell functions [17].

\section{Discrepancies between immunofluorescent and qPCR data}

After comparing results, it was clear that some of the reduction in inflammation observed in the immunofluorescent data was not reflected in the RT-qPCR data. The extent of this incongruence is difficult to gauge, as only three inflammatory markers (TNF- $\alpha$, NF$\kappa \mathrm{B}$, and MAPK3) overlapped between the two experiments. Despite this, the reduction of inflammation as a result of RI that was observed in the immunofluorescent data was not observed (in a statistically significant manner) in any of the three RT-qPCR markers. While this lack of correlation initially seems problematic, there is strong evidence that transcript levels are not always representative of protein levels. Several factors could play a part in this, including the rates of transcription, mRNA decay, translation, and protein degradation. These are all key factors that can influence the connection between measured protein and mRNA levels [18]. These incongruence are best explained by a study performing global mRNA analysis combined with mass spec data for over 5,400 proteins, where Spearman correlation coefficients of $\sim 0.42$ were obtained that indicated a poor correlation between mRNA and protein levels. This is likely due to the timeline that was used to conduct the experiment. Initial times were optimized using fluorescent microscopy, hence optimized for optimal changes in protein expression. It is likely that the delicate mRNA molecules created as a result of LPS stimulation had already degraded by the time the RT-qPCR data was collected.

\section{Final Conclusions}

In summary, the results from this study have demonstrated that the addition of the RI product to HT-29 cells results in the resolution of pro-inflammatory cytokines induced by exposure to LPS. At the protein level, these findings suggest that the RI product is capable of acting as an effective anti-inflammatory therapy to reduce cellular inflammation. Data from $\mathrm{qPCR}$ arrays indicate that a possible mechanism for the resolution of pro-inflammatory mediators may lie in alterations to the synthesis and receptor expression of prostaglandins. Given the wide-range of anti-inflammatory effects that have been attributed to prostaglandins, more research is needed to fully elucidate the mechanism of action behind the reduction of pro-inflammatory mediators observed in this study. Additionally, the patterns observed in protein and transcript expression analysis largely fit with reported studies examining the effects of zinc and ginger supplementation, both of which are included in the formulation of RI. Given that protein expression data from filtered vs. unfiltered RI both resulted in the resolution of pro-inflammatory proteins, it can be hypothesized that the large probiotic load in the product is not attributing to any inflammatory effects at the cellular level. Thus, the observations made in this study can be attributed to the zinc oxide and ginger extract in the product. More studies are necessary to further explore the synergistic effects of ginger and zinc, but this work serves as a foundation for further research and supports the findings of other research as to the anti-inflammatory effects of ginger extract and zinc oxide.

\section{References}

1. Heneka MT, Carson MJ, El Khoury J, Landreth GE, Brosseron F, et al. (2015) Neuroinflammation in Alzheimer's disease. Lancet Neurol 14: 388-405.

2. Secher T, Gaillot O, Ryffel B, Chamaillard M (2010) Remote control of intestinal tumorigenesis by innate immunity. Cancer Res 70: 1749-1752.

3. Bozimowski G (2015) A review of nonsteroidal anti-inflammatory drugs. AANA J 83: 425-433.

4. Bode AM, Dong Z (2011) The amazing and mighty ginger. In: Benzie IFF, Wachtel-Galor S (Eds) Herbal medicine: biomolecular and clinical aspects. ( $\left.2^{\text {nd }} e d n\right)$, Taylor and Francis, CRC Press.

5. Srivastava KC, Mustafa T (1992) Ginger (Zingiber officinale) in rheumatism and musculoskeletal disorders. Med Hypotheses 39: 342-348.

6. Justo OR, Simioni PU, Gabriel DL, Tamashiro WM, Rosa Pde T, et al. (2015) Evaluation of in vitro anti-inflammatory effects of crude ginger and rosemary extracts obtained through supercritical $\mathrm{CO} 2$ extraction on macrophage and tumor cell line: the influence of vehicle type. BMC Complement Altern Med 15: 390 .

7. Prasad AS (2014) Zinc is an antioxidant and anti-inflammatory agent: its role in human health. Front Nutr 1: 14

8. Reginster JY, Gillot V, Bruyere O, Henrotin Y (2000) Evidence of nutriceutical effectiveness in the treatment of osteoarthritis. Curr Rheumatol Rep 2: 472477.

9. Frondoza CG, Sohrabi A, Polotsky A, Phan PV, Hungerford DS, et al. (2004) An in vitro screening assay for inhibitors of proinflammatory mediators in herbal extracts using human synoviocyte cultures. In Vitro Cell Dev Biol Anim 40: 95-101

10. Tripathi S, Bruch D, Kittur DS (2008) Ginger extract inhibits LPS induced macrophage activation and function. BMC Complement Altern Med 8: 1

11. Lee TY, Lee KC, Chen SY, Chang HH (2009) 6-Gingerol inhibits ROS and iNOS through the suppression of PKC-alpha and NF-kappaB pathways in lipopolysaccharide-stimulated mouse macrophages. Biochem Biophys Res Commun 382: 134-139.

12. Kosaka T, Miyata A, Ihara H, Hara S, Sugimoto T, et al. (1994) Characterization of the human gene (PTGS2) encoding prostaglandin-endoperoxide synthase 2. Eur J Biochem 221: 889-897.

13. Torres-Atencio I, Ainsua-Enrich E, de Mora F, Picado C, Martín M (2014) Prostaglandin E2 prevents hyperosmolar-induced human mast cell activation through prostanoid receptors EP2 and EP4. PLoS One 9: e110870.

14. Tjendraputra E, Tran VH, Liu-Brennan D, Roufogalis BD, Duke CC (2001) 
Citation: Norton R, O’Reilly A, Miley D. Analysis of Gene Expression in a Gut Epithelial Cell Line Treated with re:iimmune and Lipopolysaccharide. J Nutri Health. 2016;2(2): 6.

ISSN: $2469-4185$

Effect of ginger constituents and synthetic analogues on cyclooxygenase-2 enzyme in intact cells. Bioorg Chem 29: 156-163.

15. Song MK, Adham NF (1985) Relationship between zinc and prostaglandin metabolisms in plasma and small intestine of rats. Am J Clin Nutr 41: 1201 1209.

16. Ricciotti E, FitzGerald GA (2011) Prostaglandins and inflammation Arterioscler Thromb Vasc Biol 31: 986-1000
17. Harris SG, Padilla J, Koumas L, Ray D, Phipps RP (2002) Prostaglandins as modulators of immunity. Trends Immunol 23: 144-150.

18. Vogel C, Abreu Rde S, Ko D, Le Sy, Shapiro BA, et al. (2010) Sequence signatures and mRNA concentration can explain two-thirds of protein abundance variation in a human cell line. Mol Syst Biol 6: 400.

\section{Acknowledgements}

This work was funded in-full by Make People Better, LLC. 